\title{
NIVEAUX DE SPIN ÉLEVÉ DANS LE NOYAU ${ }^{64} \mathrm{Zn}$
}

\author{
J. F. BRUANDET, F. GLASSER, M. AGARD, A. GIORNI, J. P. LONGEQUEUE \\ et TSAN UNG CHAN
}

\author{
Institut des Sciences Nucléaires, BP 257, 38044 Grenoble Cedex, France
}

(Reçu le 2 décembre 1975, révisé le 16 janvier 1976, accepté le 9 février 1976)

\begin{abstract}
Résumé. - Un schéma de niveaux a été obtenu pour le noyau ${ }^{64} \mathrm{Zn}$, en spectroscopie $\gamma$ sur faisceau, par l'étude de la réaction ${ }^{62} \mathrm{Ni}(\alpha, 2 \mathrm{n} \gamma)$. Les caractéristiques $\mathrm{J}^{\pi}=6^{+}$et $7^{(-)}$sont proposées pour les niveaux à $4236 \mathrm{keV}$ et $4635 \mathrm{keV}$ respectivement. La vie moyenne $(\tau=130 \pm 15 \mathrm{ps})$ de la transition $E_{\gamma}=641 \mathrm{keV}$ désexcitant le niveau à $4635 \mathrm{keV}$ a été mesurée par la méthode du parcours de recul dans la réaction ${ }^{55} \mathrm{Mn}\left({ }^{12} \mathrm{C}, \mathrm{p} 2 \mathrm{n} \gamma\right)$.

Abstract. - A level scheme of the ${ }^{64} \mathrm{Zn}$ nucleus has been established from the study of the ${ }^{62} \mathrm{Ni}(\alpha, 2 \mathrm{n} \gamma)$ reaction by in-beam gamma spectroscopy. We propose the quantum numbers $\mathrm{J}^{\pi}=6^{+}$ for the $4236 \mathrm{keV}$ level and $\mathrm{J}^{\pi}=7^{(-)}$for the $4635 \mathrm{keV}$ level. The mean life ( $\tau=130 \pm 15 \mathrm{ps}$ ) of the $641 \mathrm{keV} \gamma$-ray de-exciting the $4635 \mathrm{keV}$ level has been measured by the recoil-distance method using the ${ }^{55} \mathrm{Mn}\left({ }^{12} \mathrm{C}, \mathrm{p} 2 \mathrm{n} \gamma\right)$ reaction.
\end{abstract}

1. Introduction. - Nous avons entrepris l'étude systématique des isotopes pairs des zincs par spectroscopie $\gamma[1,2]$. La couche de neutrons $1_{g_{9 / 2}}$ est suffisamment voisine de la couche $1 f_{5 / 2}$ [3] pour que l'on puisse espérer mettre en évidence, dans ces noyaux, des niveaux de spin élevé à une énergie d'excitation de quelques MeV. Dans ce but, nous avons étudié pour plusieurs énergies incidentes, la formation du noyau de ${ }^{64} \mathrm{Zn}$ par les réactions ${ }^{54} \mathrm{Fe}\left({ }^{12} \mathrm{C}, 2 \mathrm{p} \gamma\right){ }^{64} \mathrm{Zn}$ $\left(E_{12 \mathrm{C}}=36\right.$ à $\left.48 \mathrm{MeV}\right)$ et ${ }^{62} \mathrm{Ni}(\alpha, 2 \mathrm{n} \gamma)^{64} \mathrm{Zn}\left(E_{\alpha}=22\right.$ à $35 \mathrm{MeV}$ ) : l'expérience a montré que dans ces deux réactions les mêmes niveaux étaient atteints avec des intensités d'alimentation du même ordre [1]. Nous présentons ici essentiellement la cascade $\gamma$ la plus intense ainsi que le résultat d'une mesure de durée de vie par la méthode du parcours de recul.

2. Spectroscopie du noyau ${ }^{64} \mathrm{Zn}$ par la réaction ${ }^{62} \mathrm{Ni}(\alpha, 2 \mathrm{n} \gamma)$. - Nous avons utilisé les faisceaux de particules $\alpha$ accélérées par le cyclotron de Grenoble, une cible autoporteuse de ${ }^{62} \mathrm{Ni}\left(700 \mu \mathrm{g} / \mathrm{cm}^{2}\right)$ enrichie à $99,4 \%$ et des détecteurs $\mathrm{Ge}(\mathrm{Li})\left(30\right.$ à $\left.60 \mathrm{~cm}^{3}\right)$ ayant une résolution typique de $3 \mathrm{keV}$ à $1,33 \mathrm{MeV}$. Quatre types de mesure ont été effectués : 1) Des coïncidences $\gamma-\gamma$ promptes et retardées [4] à l'énergie $E_{\alpha}=31 \mathrm{MeV}$ : la figure 1 montre le spectre de coincidences totales $\gamma-\gamma$ et quelques spectres en coïncidence avec des raies $\gamma$ du ${ }^{64} \mathrm{Zn}$. 2) Des fonctions d'excitation pour des énergies $E_{\alpha}$ variant de 22 à $40 \mathrm{MeV}$ (Fig. 2) : ces fonctions croissent avec l'énergie $E_{\alpha}$ d'autant plus fortement que la raie $\gamma$ considérée correspond à la désexcitation d'un niveau de spin d'autant plus élevé.
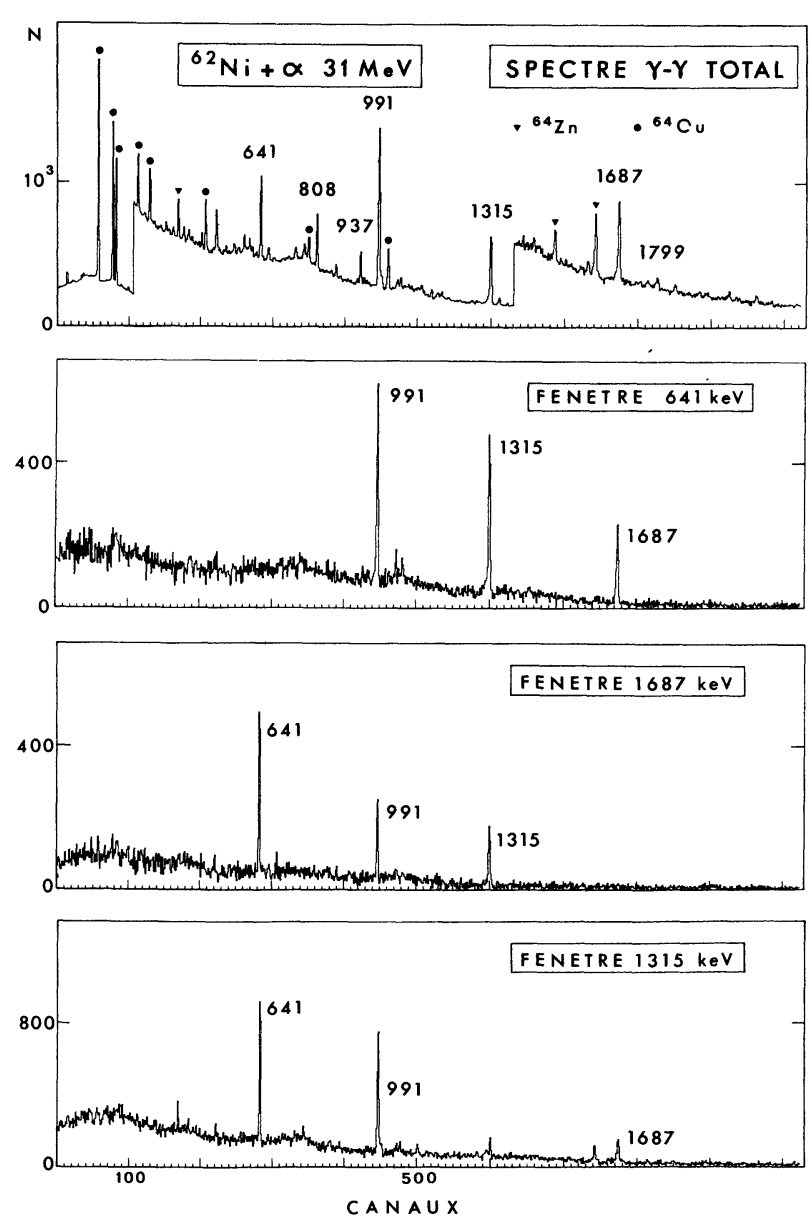

FIG. 1. - Spectres de coïncidences $\gamma-\gamma$ obtenus dans la réaction ${ }^{62} \mathrm{Ni}+\alpha$ à $E_{\alpha}=31 \mathrm{MeV}$. 
TABLEAU I

Résultats des distributions angulaires dans ${ }^{64} \mathrm{Zn}$

\begin{tabular}{|c|c|c|c|c|c|}
\hline \multicolumn{3}{|c|}{ Transition } & \multicolumn{2}{|c|}{ Coefficients de distribution angulaire $\left(^{a}\right)$} & Mélange $\delta\left({ }^{b}\right)$ \\
\hline$E_{\gamma}$ & $\mathbf{J}_{\mathbf{i}}$ & $\mathbf{J}_{\mathbf{f}}$ & $A_{2} \pm \Delta A_{2}$ & $A_{4} \pm \Delta A_{4}$ & Multipolarité \\
\hline 991 & $\overline{2^{+}}$ & $\overline{0^{+}}$ & $0,177 \pm 0,015$ & $-0,121 \pm 0,022$ & $\mathrm{E}_{2} \overline{\text { pure }}$ \\
\hline 1315 & $4^{+}$ & $2^{+}$ & $0,280 \pm 0,022$ & $-0,123 \pm 0,033$ & $E_{2}$ pure \\
\hline 1687 & $6^{+}$ & $4^{+}$ & $0,345 \pm 0,029$ & $-0,174 \pm 0,043$ & $E_{2}$ pure \\
\hline 641 & $7^{-}$ & $6^{+}$ & $-0,343 \pm 0,026$ & $-0,071 \pm 0,037$ & $L=1$ pure \\
\hline 808 & $2^{+}$ & $2^{+}$ & $-0,216 \pm 0,026$ & $-0,071 \pm 0,037$ & $\begin{array}{r}-0,57^{-0,27}+0,13 \\
\end{array}$ \\
\hline 937 & $4^{+}$ & $2^{+}$ & $0,263 \pm 0,015$ & $-0,135 \pm 0,023$ & $\mathrm{E}_{2}$ pure \\
\hline
\end{tabular}

$\left({ }^{a}\right) W(\theta)=1+A_{2} P_{2}(\cos \theta)+A_{4} P_{4}(\cos \theta)$.

$\left({ }^{b}\right)$ Erreur sur $\delta$ déterminée en considérant un écart standard de variation par rapport à $\chi_{\min }^{2}$.

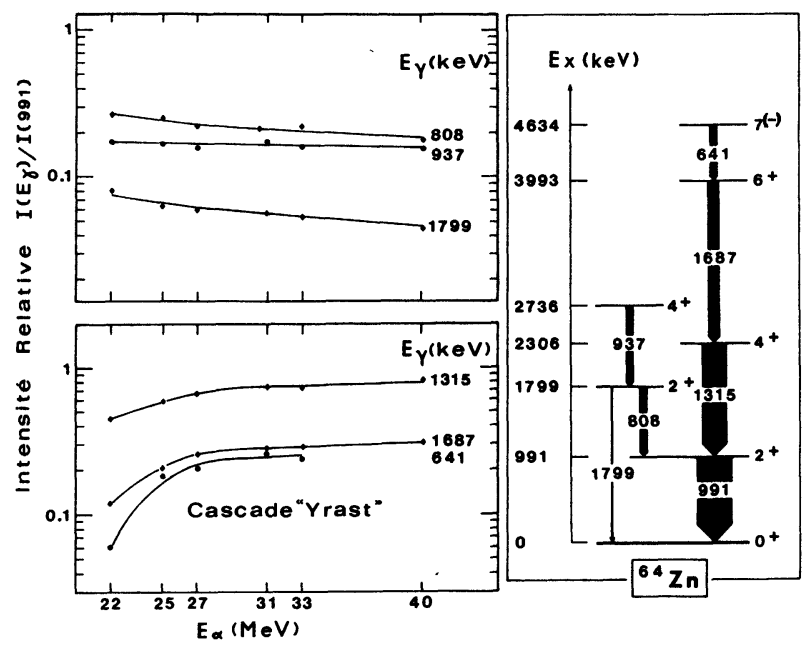

Fig. 2. - Fonctions d'excitation relatives de quelques transitions $\gamma$ dans le noyau ${ }^{64} \mathrm{Zn}$ et schéma de niveaux partiel.

3) Des distributions angulaires $\gamma$ à $E_{\alpha}=31 \mathrm{MeV}$ dont l'analyse est résumée dans le tableau I. 4) Une détection des raies $\gamma$ retardées par observation du spectre $\gamma$ dans l'intervalle de temps (70 ns) compris entre deux impulsions de faisceau : aucune raie $\gamma$ retardée de période $>5$ ns n'a été mise en évidence dans le noyau ${ }^{64} \mathrm{Zn}$.

3. Mesure des périodes de décroissance des raies $y_{0}$ Cette mesure a été effectuée par la méthode du parcours de recul basée sur l'utilisation de l'effet Doppler [5]. Les réactions avec ions lourds sont particulièrement adaptées à ce type de mesures : ayant eu des difficultés pour réaliser une cible très plane de ${ }^{54} \mathrm{Fe}$, nous avons remplacé la réaction ${ }^{54} \mathrm{Fe}\left({ }^{12} \mathrm{C}, 2 \mathrm{p} \gamma\right)$ par la réaction ${ }^{55} \mathrm{Mn}\left({ }^{12} \mathrm{C}, \mathrm{p} 2 \mathrm{n} \gamma\right)$ à $E_{12 \mathrm{C}}=35 \mathrm{MeV}$ (cible de $100 \mu \mathrm{g} / \mathrm{cm}^{2}$ de ${ }^{55} \mathrm{Mn}$, déposée sur un support d'or de $1 \mathrm{mg} / \mathrm{cm}^{2}$ ). L'appareillage [6] est constitué d'un stoppeur en tungstène dont la surface a une planéité meilleure que $0,1 \mu$ sur un diamètre de $10 \mathrm{~mm}$ et d'un chariot portant la cible, dont la translation autorise des déplacements de 0 à $4 \mathrm{~mm}$ avec une sensibilité de position de $0,5 \mu$. Le système de tension de la cible est tel que les défauts de planéité n'excèdent pas $0,5 \mu$ et le réglage du parallélisme des plans de la cible et du stoppeur est réalisé par une méthode optique d'autocollimation avec une précision angulaire meilleure que $10^{\prime \prime}$. La mesure de la distance cible-stoppeur est effectuée à l'aide d'un

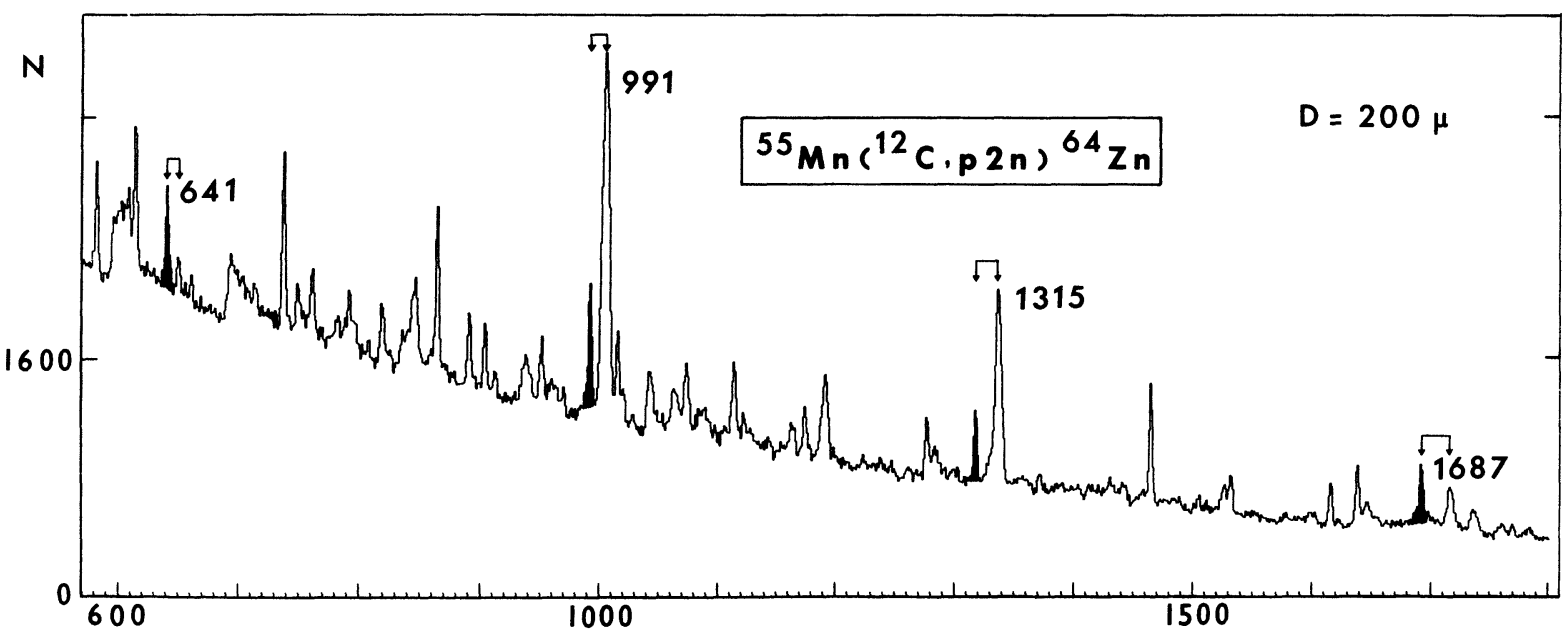

C A NAUX

Fig. 3. - Spectre $\gamma$ libre obtenu dans la réaction ${ }^{55} \mathrm{Mn}+{ }^{12} \mathrm{C}$ à $E_{12 \mathrm{C}}=35 \mathrm{MeV}$. 

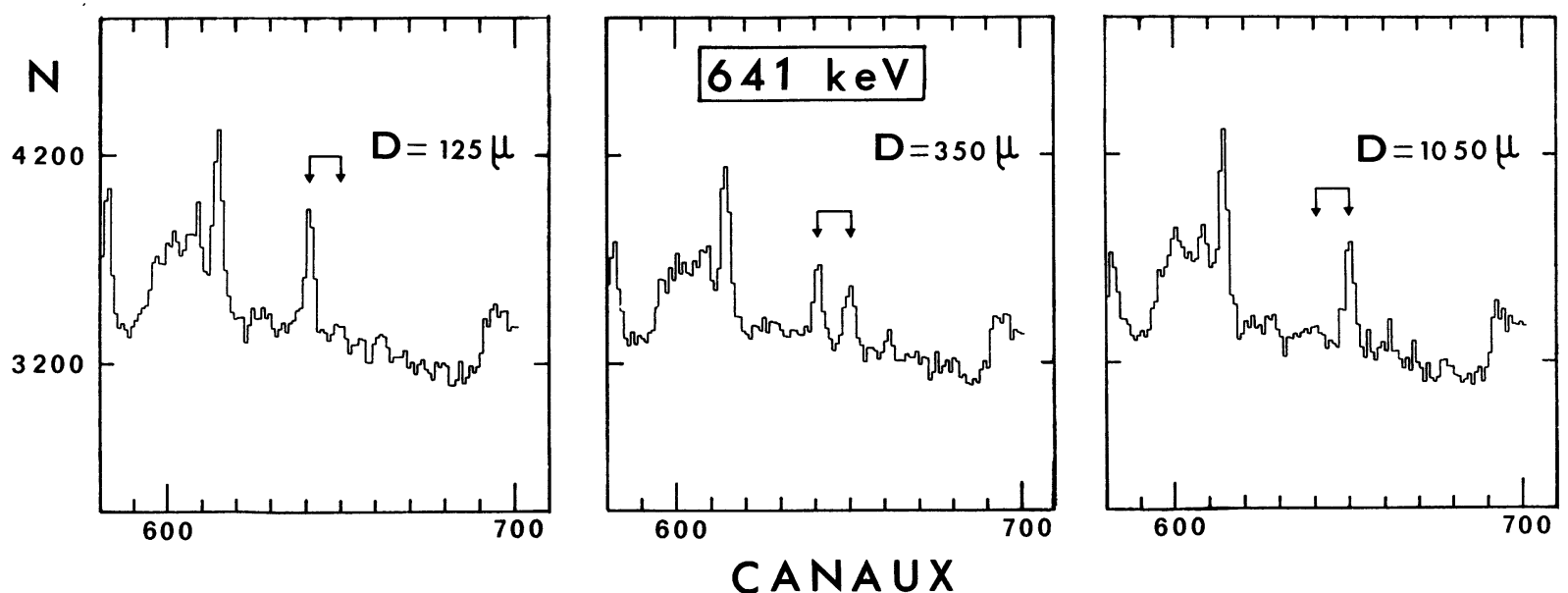

Fig. 4. - Echantillons de spectres $\gamma$ de la réaction ${ }^{55} \mathrm{Mn}\left({ }^{12} \mathrm{C}, \mathrm{p} 2 \mathrm{n}\right)$ à $35 \mathrm{MeV}$ montrant le déplacement de la raie $E_{\gamma}=641 \mathrm{keV}$ en fonction de la distance cible-stoppeur.

comparateur ayant une précision de $1 \mu$; afin de connaître avec précision la distance zéro et pour contrôler les variations de cette distance, une méthode de mesure capacitive a été utilisée. Cet appareillage [6] a été testé sur des mesures de durée de vie dans ${ }^{41} \mathrm{Ca}$ et ${ }^{41} \mathrm{~K}$, obtenus par réactions ${ }^{16} \mathrm{O}+{ }^{27} \mathrm{Al}$, publiées récemment [7]. La figure 3 représente l'ensemble du spectre $\gamma$ enregistré à $0^{\circ}$ par rapport au faisceau pour une distance cible-stoppeur $D=200 \mu$, et la figure 4 montre le déplacement Doppler de la raie $E_{\gamma}=641 \mathrm{keV}$ (dans ${ }^{64} \mathrm{Zn}$ ) pour différentes distances $D$. La courbe de décroissance de cette raie est présentée sur la figure 5 : elle correspond à une vie moyenne

$$
\tau=130 \pm 15 \mathrm{ps},
$$

calculée à partir de la relation

$$
\frac{I_{0}}{I_{0}+I_{\mathrm{s}}}=\mathrm{e}^{-D / v \tau}
$$
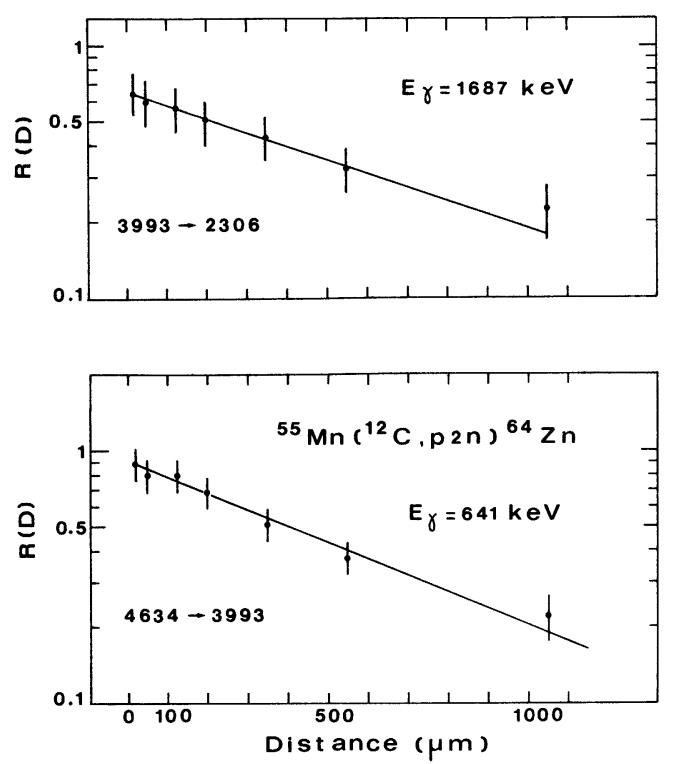

Fig. 5. - Intensité relative $R(D)=\frac{I_{0}}{I_{0}+I_{\mathrm{s}}}$ du pic non déplacé pour chacune des raies $E_{\gamma}=1687 \mathrm{keV}$ et $E_{\gamma}=641 \mathrm{keV}$. dans laquelle $I_{\mathrm{s}}$ et $I_{0}$ sont respectivement les intensités de la raie $\gamma$ déplacée (émise par les noyaux excités de ${ }^{64} \mathrm{Zn}$ reculant dans le vide avec la vitesse $v$ ) et de la raie $\gamma$ non déplacée (émise par les noyaux de ${ }^{64} \mathrm{Zn}$ arrêtés dans le stoppeur). L'allure de cette courbe de décroissance montre que les périodes des transitions $\gamma$ alimentant le niveau à $4635 \mathrm{keV}$ sont négligeables devant la période de cet état. Nous avons d'ailleurs $\mathrm{pu}$ vérifier expérimentalement que la principale transition, d'énergie $E_{\gamma}=1046 \mathrm{keV}$, alimentant ce niveau a une durée de vie beaucoup plus faible.

De la courbe de décroissance (Fig. 5) de la transition $E_{\gamma}=1687 \mathrm{keV}$, nous avons déduit, après soustraction de la contribution due à la raie de $641 \mathrm{keV}$ [5], une limite supérieure $\tau \leqslant 20$ ps pour la vie moyenne du niveau à $3993 \mathrm{keV}$.

4. Discussion des résultats. - Nos résultats sont résumés sur la figure 2 . Les niveaux à $991 \mathrm{keV}$ et $2306 \mathrm{keV}$ sont connus [8] comme ayant les caractéristiques $\mathrm{J}^{\pi}=2^{+}$et $4^{+}$respectivement. Pour le niveau à $3993 \mathrm{keV}$, nous avons proposé [1] les caractéristiques $\mathrm{J}^{\pi}=6^{+}$sur la base des considérations suivantes : 1) L'analyse de la distribution angulaire et la croissance de la fonction d'excitation, pour la raie $\gamma$ de $1687 \mathrm{keV}$, ainsi que l'absence de transitions $\gamma$ vers les niveaux $0^{+}$et $2^{+}$à plus basse énergie, sont en faveur d'un spin $J=6$. 2) La limite supérieure de la vie moyenne ( $\tau<20 \mathrm{ps}$ ) de la raie $\gamma$ de $1686 \mathrm{keV}$, exclut la caractéristique M2 pour cette transition [9], c'est-à-dire exclut une parité négative pour ce niveau. Ce résultat a été confirmé par R. O. Sayer et al. [10] qui ont étudié le noyau ${ }^{64} \mathrm{Zn}$ par la réaction ${ }^{51} \mathrm{~V}\left({ }^{16} \mathrm{O}, \mathrm{p} 2 \mathrm{n} \gamma\right)$ et ont proposé $\mathrm{J}^{\pi}=6^{(+)}$pour ce niveau. Pour le niveau à $4635 \mathrm{keV}$, nous proposons avec les mêmes arguments que précédemment la caractéristique $J=7$. La mesure de la vie moyenne de la raie $E_{y}=641 \mathrm{keV}(\tau=130 \pm 15 \mathrm{ps})$ conduit dans l'hypothèse d'une transition $\mathrm{M} 1$ à une intensité de $10^{-3}$ unité Weisskopf, c'est-à-dire à une valeur 
extrême du facteur de retard pour ce type de transition [11]; nous proposons donc de considérer qu'il s'agit d'une transition E1 et d'attribuer une parité négative au niveau à $4635 \mathrm{keV}$, c'est-à-dire les caractéristiques $\mathrm{J}^{\pi}=7^{-}$. Une interprétation théorique élémentaire de ce niveau (analogue à celles que nous avons faites pour le noyau ${ }^{66} \mathrm{Zn}$ [2]) consiste à consi- dérer qu'il s'agit de la configuration $\left[1 \mathrm{f}_{5 / 2} \cdot 1 \mathrm{~g}_{9 / 2}\right]^{\mathrm{J}_{\pi}=7-}$ en neutrons.

Remerciements. - Nous remercions $M$. le Pr. A. Gallmann et son équipe pour les conseils qu'ils nous ont donnés dans la réalisation de notre appareillage de mesure de durée de vie.

\section{Bibliographie}

[1] Bruandet, J.-F., TSan Ung Chan, Longequeue, J.-P., Agard, M. et Giorni, A., J. Physique Colloq. 35 (1974) C5-2; Proc. Int. Symp. on Highly Excited States in Nuclei, Jülich (1975), vol. 1, p. 40.

[2] Bruandet, J.-F., Agard, M., GioRni, A., Longequeue, J.-P., Morand, C. et Tsan Ung Chan, à paraitre dans Phys. Rev. (Dec. 1975)

[3] Von Ehrenstein, D. and Schiffer, J.-P., Phys. Rev. 164 (1967) 1374.

[4] Tsan Ung Chan, Agard, M., Bruandet, J.-F., Giorni, A. and LongeQueue, J.-P., à paraître dans Nucl. Phys.

[5] Nuclear Spectroscopy and Reactions, Part C. Edited by Cerny, J. (Academic Press) 1974, p. 341.
[6] GLASSER, F., Thèse 3e cycle, Université de Grenoble (1975).

[7] LieB, K. P., Uhrmacher, M., Dauk, J. and Kleinfeld, A. M., Nucl. Phys. A 223 (1974) 445.

[8] Auble, R. L., Nucl. Data Sheets 12 (1974) 305.

[9] Letessier, J. et Foucher, R., Ann. Phys. (Paris) 4 (1969) 55.

[10] SAYer, R. O., Robinson, R. L., MiLneR, W. T. and SMith, G. J., Int. Conf. on Reactions between Complex Nuclei, Nashville, vol. 1 (Amsterdam, North-Holland) 1974, p. 192.

[11] Skorka, S. J., Hertel, J. and RetZ-SchmidT, J. W., Nuclear Data, Sect. A 2 (1966) no 4.

ENDT, P. M. and VAN DER LeUn, C., Nucl. Data Tables 13 (1974) 67. 